\title{
A phase II randomized trial of Observation versus stereotactic ablative Radiatlon for OLigometastatic prostate CancEr (ORIOLE).
}

\author{
Noura Radwan \\ Johns Hopkins University School of Medicine \\ Ryan Phillips \\ Johns Hopkins University School of Medicine \\ Ashley Ross \\ Johns Hopkins University School of Medicine \\ Steven P. Rowe

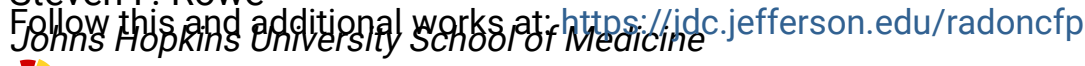 \\ Part of the Oncology Commons, and the Radiology Commons \\ MichaelA. Gorin
}

\section{Recommended Citation \\ Radwan, Noura; Phillips, Ryan; Ross, Ashley; Rowe, Steven P.; Gorin, Michael A.; Antonarakis, Emmanuel S.; Deville, Curtiland; Greco, Stephen; Denmeade, Samuel; Paller, Channing; Song, Daniel Y.; Diehn, Maximilian; Wang, Hao; Carducci, Michael; Pienta, Kenneth J.; Pomper, Martin G.; DeWeese, Theodore L.; Dicker, Adam; Eisenberger, Mario; and Tran, Phuoc T., "A phase II randomized trial of Observation versus stereotactic ablative Radiatlon for OLigometastatic prostate CancEr (ORIOLE)." (2017). Department of Radiation Oncology Faculty Papers. Paper 95. https://jdc.jefferson.edu/radoncfp/95}

This Article is brought to you for free and open access by the Jefferson Digital Commons. The Jefferson Digital Commons is a service of Thomas Jefferson University's Center for Teaching and Learning (CTL). The Commons is a showcase for Jefferson books and journals, peer-reviewed scholarly publications, unique historical collections from the University archives, and teaching tools. The Jefferson Digital Commons allows researchers and interested readers anywhere in the world to learn about and keep up to date with Jefferson scholarship. This article has been accepted for inclusion in Department of Radiation Oncology Faculty Papers by an authorized administrator of the Jefferson Digital Commons. For more information, please contact: JeffersonDigitalCommons@jefferson.edu. 


\section{Authors}

Noura Radwan, Ryan Phillips, Ashley Ross, Steven P. Rowe, Michael A. Gorin, Emmanuel S. Antonarakis, Curtiland Deville, Stephen Greco, Samuel Denmeade, Channing Paller, Daniel Y. Song, Maximilian Diehn, Hao Wang, Michael Carducci, Kenneth J. Pienta, Martin G. Pomper, Theodore L. DeWeese, Adam Dicker, Mario Eisenberger, and Phuoc T. Tran 


\title{
A phase II randomized trial of Observation versus stereotactic ablative Radiatlon for OLigometastatic prostate CancEr (ORIOLE)
}

Noura Radwan ${ }^{1 \dagger}$, Ryan Phillips ${ }^{1 \dagger}$, Ashley Ross ${ }^{2,3}$, Steven P. Rowe ${ }^{4}$, Michael A. Gorin ${ }^{3}$, Emmanuel S. Antonarakis ${ }^{2}$, Curtiland Deville ${ }^{1,2}$, Stephen Greco', Samuel Denmeade ${ }^{2,3}$, Channing Paller ${ }^{2}$, Daniel Y. Song ${ }^{1,2,3}$, Maximilian Diehn ${ }^{5}$, Hao Wang ${ }^{2}$, Michael Carducci ${ }^{2,3}$, Kenneth J. Pienta ${ }^{2,3}$, Martin G. Pomper ${ }^{1,2,3,4}$, Theodore L. DeWeese $e^{1,2,3}$, Adam Dicker ${ }^{6}$, Mario Eisenberger ${ }^{2,3+}$ and Phuoc T. Tran ${ }^{1,2,3^{*}+}$

\begin{abstract}
Background: We describe a randomized, non-blinded Phase II interventional study to assess the safety and efficacy of stereotactic ablative radiotherapy (SABR) for hormone-sensitive oligometastatic prostate adenocarcinoma, and to describe the biology of the oligometastatic state using immunologic, cellular, molecular, and functional imaging correlates. 54 men with oligometastatic prostate adenocarcinoma will be accrued. The primary clinical endpoint will be progression at 6 months from randomization with the hypothesis that SABR to all metastases will forestall progression by disrupting the metastatic process. Secondary clinical endpoints will include local control at 6 months post-SABR, toxicity and quality of life, and androgen deprivation therapy (ADT)-free survival (ADT-FS). Further fundamental analysis of the oligometastatic state with be achieved through correlation with investigational ${ }^{18}$ F-DCFPyL PET/CT imaging and measurement of circulating tumor cells, circulating tumor DNA, and circulating T-cell receptor repertoires, facilitating an unprecedented opportunity to characterize, in isolation, the effects of SABR on the dynamics of and immunologic response to oligometastatic disease.

Methods/design: Patients will be randomized 2:1 to SABR or observation with minimization to balance assignment by primary intervention, prior hormonal therapy, and PSA doubling time. Progression after 6 months will be compared using Fisher's exact test. Hazard ratios and Kaplan-Meier estimates of progression free survival (PFS), ADT free survival (ADT-FS), time to locoregional progression (TTLP) and time to distant progression (TTDP) will be calculated based on an intention-to-treat. Local control will be assessed using Response Evaluation Criteria in Solid Tumors (RECIST) 1.1 criteria. Withdrawal from the study prior to 6 months will be counted as progression. Adverse events will be summarized by type and grade. Quality of life pre- and post- SABR will be measured by Brief Pain Inventory.

* Correspondence: tranp@jhmi.edu

†Equal contributors

'Department of Radiation Oncology \& Molecular Radiation Sciences, The

Sidney Kimmel Comprehensive Cancer Center, Johns Hopkins University

School of Medicine, 1550 Orleans Street, CRB2 Rm 406, Baltimore, MD 21231,

USA

2Department of Medical Oncology, The Sidney Kimmel Comprehensive

Cancer Center, Johns Hopkins University School of Medicine, Baltimore, MD,

USA

Full list of author information is available at the end of the article 


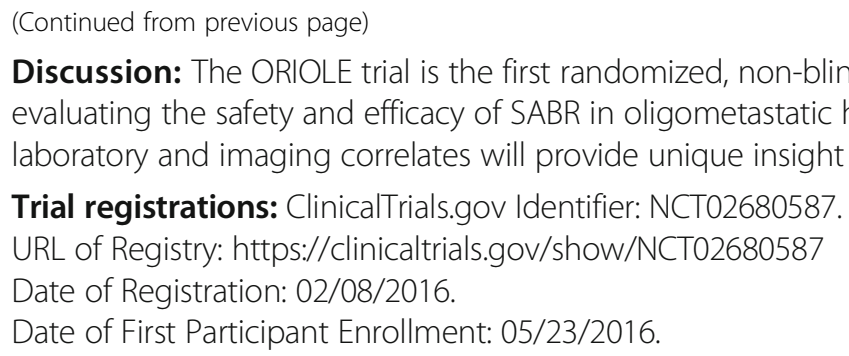

Trial registrations: ClinicalTrials.gov Identifier: NCT02680587.

URL of Registry: https://clinicaltrials.gov/show/NCT02680587

Date of Registration: 02/08/2016.

Date of First Participant Enrollment: 05/23/2016.

Discussion: The ORIOLE trial is the first randomized, non-blinded Phase II interventional study in the North America evaluating the safety and efficacy of SABR in oligometastatic hormone-sensitive prostate cancer. Leading-edge laboratory and imaging correlates will provide unique insight into the effects of SABR on the oligometastatic state.

Keywords: Prostate cancer, Stereotactic body radiation therapy, Stereotactic ablative radiotherapy, Oligometastasis

\section{Background}

Cancer is the second leading cause of death in the United States, chiefly from an inability to control metastatic disease. Systemic therapy alone is not curative for patients with most metastatic solid tumors [1]. The metastatic capacity of cancers behaves along a spectrum of disease progression, such that some tumors have spread widely before clinical detectability and others rarely if ever metastasize. The presence of an oligometastatic state, at which point metastases are limited in number and location, was originally proposed by Hellman and Weichselbaum, who suggested that these patients would benefit from effective local therapy in addition to systemic therapy [1].

The treatment of metastases depends on multiple factors including 1) the location of the primary tumor, 2) the size, number and location of metastases, 3) the availability and effectiveness of therapies (e.g. surgery, radiation, and chemotherapy), and 4) the patient's functional status. Conventional moderate dose radiation for metastatic disease is given primarily for palliation, but recent advances in radiation delivery now make it possible to image and treat precisely within any anatomic region of the body [2, 3]. As a result, highly accurate radiation at tumorocidal doses can be delivered in 1 to 5 outpatient treatments [4-8].

Stereotactic radiation therapy entails highly conformal and precisely targeted radiation delivered in a very dose intensive fashion. In the brain, this approach (termed stereotactic radiosurgery or SRS) has been shown to be a highly effective treatment for brain metastases [9]. Data suggests that select small extracranial tumors (either primary or metastatic tumors) may be effectively controlled using a similar approach known as stereotactic body radiotherapy (SBRT) or stereotactic ablative radiotherapy (SABR). Local control in excess of $75 \%$ has been reported for metastatic tumors of the spine, lung and liver, which is significantly higher than standard conventional moderate dose radiation $[5,7,8,10-23]$. Toxicity has been minimal in multiple U.S., European, and Japanese trials of SABR to the lung, liver, spine, pelvis and abdomen despite the use of very high biological equivalent doses for patients with both organ-confined and metastatic cancer.
The natural history of hormone sensitive oligometastatic prostate cancer is under studied. However, much is known regarding the preceding state of biochemically recurrent prostate cancer that has failed primary treatment. The management of this heterogeneous group of men with a rising PSA often involves relatively long periods of observation until metastases develop at which time the initiation of androgen deprivation therapy (ADT) is typically recommended. Although not entirely appropriate for all men with biochemical failure, data would suggest stalling initiation of ADT is not likely overtly detrimental to overall survival [24]. In the modern era with conventional imaging, oligometastatic hormone sensitive prostate cancer likely comprises a large number of men, possibly the majority of men following failed primary therapy [25-28]. Assuming these men are at a potentially curable state before castration-resistance develops, we need additional treatment strategies to re-examine this large cohort of men.

Based on this emerging evidence, we propose a phase II study of SABR in patients with oligometastatic hormone sensitive prostate cancer. This study is designed to determine if we can improve the outcome of prostate cancer in these men and also to advance the basic understanding of the oligometastatic state as it pertains to signaling dynamics, cell biology, and immunologic responses. Clinically, we anticipate that SABR in the oligometastatic setting will safely forestall disease progression, thereby lengthening the time before initiation of hormonal therapy and protecting patients from the known deleterious side effects of this conventional systemic approach and thus improve quality of life [24].

As we continue to refine the standard approaches to treatment of oligometastatic cancer within and beyond the prostate, principle questions remain unanswered which may greatly enhance our collective ability to improve patient outcomes. Chief among these are how best to identify patients in the oligometastatic state, and what aspects of this state differentiate it fundamentally from patients with organ-confined or polymetastatic disease. To address the former, PET/CT imaging utilizing the investigational prostate specific membrane antigen (PMSA) targeted 
radiotracer, ${ }^{18} \mathrm{~F}$-DCFPyL, will be compared to conventional bone scan and CT imaging to assess the utility of this imaging test in identifying oligometastases before SABR and monitoring disease response following SABR [29-31]. Alterations in the biology of the oligometastatic state induced by SABR will be investigated using leading-edge correlatives, including: analysis of circulating tumor cells (CTCs; Epic Sciences, San Diego, CA), deep sequencing of circulating tumor DNA (ctDNA) using Cancer Personalized Profiling by deep sequencing (CAPP-Seq) to non-invasively assess tumor burden, and ImmunoSEQ profiling of T-cell repertoires to elucidate the immunological response to SABR (Adaptive Technologies, Seattle, WA). Finally, use of the Color Genomics platform (Burlingame, CA), a hereditary cancer assay assessing pathogenic mutations in 30 cancer predisposition genes that account for $>90 \%$ of the germline mutations known to occur in men with castrate resistant metastatic prostate cancer (mCRPC), will inform efforts to advance a more personalized medicine approach to tailor screening and therapies to these men $[32,33]$.

\section{Methods/design}

This study was approved by the Reaserach Ethics Boards of Johns Hopkins Medicine. The ORIOLE Trial is registered at the US National Institutes of Health (ClinicalTrials.gov) \# NCT02680587 and Current Controlled Trials IND/IDE Number: 121064.

\section{Objectives}

Primary endpoint: to determine the proportion of men with oligometastatic hormone sensitive prostate cancer who have progressed after 6 months from randomization to observation versus SABR.

\section{Secondary endpoints}

- To describe the toxicity of SBRT/SABR delivered for the population enrolled using Common Terminology Criteria for Adverse Events (CTCAE) version 4.0.

- To determine local control at 6-months after SABR in patients with oligometastatic disease.

- To assess progression free survival (PFS) and ADTfree survival (ADT-FS) after randomization defined as the time interval between the day of randomization and progression.

- To assess quality of life in the SBRT/SABR arm using the Brief Pain Inventory form [34].

- To estimate the proportion of ${ }^{18} \mathrm{~F}$-DCFPyL-PET/MRI or $-\mathrm{PET} / \mathrm{CT}$ positive sites that are positive for new or progressive metastatic disease by bone scan/CT at baseline and 6 months following SABR and vice versa.

- To enumerate CTCs using EPIC HD-CTC platforms at baseline and day 180 from randomization.
- To enumerate circulating tumor DNA (ctDNA) using Cancer Personalized Profiling by deep sequencing (CAPP-Seq) at baseline, day 90 and day 180 from randomization for control and SABR arms.

- To quantitatively sequence T-cell receptor (TCR) repertoires using peripheral blood monocytes and the ImmunoSEQ platform at baseline and day 90 from randomization.

\section{Inclusion criteria}

- Patient must have 1-3 asypmtomatic metastatic tumor(s) of the bone or soft tissue developed within the past 6-months that are $\leq 5.0 \mathrm{~cm}$ or $<250 \mathrm{~cm}^{3}$.

- Patient must have had their primary tumor treated with surgery and/or radiation and salvage radiation to the prostate bed or pelvis is allowed.

- Histologic confirmation of malignancy (primary or metastatic tumor).

- Prostate specific antigen (PSA) $\geq 0.5 \mathrm{ng} / \mathrm{mL}$ but $\leq$ $50 \mathrm{ng} / \mathrm{mL}$ and Testosterone $\geq 125 \mathrm{ng} / \mathrm{dL}$.

- PSA doubling time (PSADT) $<15$ months. PSADT will be calculated using as many PSA values that are available from time of relapse (PSA > $0.2 \mathrm{ng} / \mathrm{dL}$ ).

- Patient may have had prior systemic therapy and/or ADT associated with treatment of their primary prostate cancer. Patient may have had ADT associated with salvage radiation therapy.

- Patient must be $\geq 18$ years of age, have the ability to understand, and the willingness to sign, a written informed consent document.

- Patient must have an Eastern Cooperative Oncology Group performance status $\leq 2$.

- Patient must have normal organ and marrow function as defined as:

- Leukocytes $\geq 2,000 / \mu \mathrm{L}$, absolute neutrophil count $\geq 1,000 / \mu \mathrm{L}$, platelets $\geq 50,000 / \mu \mathrm{L}$

\section{Exclusion criteria}

- No more than 3 years of ADT is allowed, with the most recent ADT treatment having occurred greater than 6 months prior to enrollment.

- ${ }^{18}$ F-DCFPyL-PET/MRI or ${ }^{18}$ F-DCFPyL-PET/CT scan within the past 6 months with results that demonstrate lesions not seen on baseline $\mathrm{CT}$ or bone scan

- Castration-resistant prostate cancer (CRPC).

- Spinal cord compression or impending spinal cord compression.

- Suspected pulmonary and/or liver metastases (greater $\geq 10 \mathrm{~mm}$ in largest axis).

- Receipt of any other investigational agents or participation in a concurrent treatment protocol. 
- Serum creatinine and total bilirubin $>3$ times the upper limit of normal

- Liver Transaminases $>5$-times the upper limit of normal.

- Inability to lie flat during or tolerate PET/CT, PET/MRI or SABR.

- Refusal to sign informed consent.

\section{Evaluation of randomization and blinding}

This study will employ a randomized phase II design to determine the appropriateness of a subsequent phase III trial based on comparison of rate of progression at 6 months. An interactive web response system (IWRS) will be utilized to obtain the patient's randomization assignment. Randomization will occur in a 2:1 fashion for SABR and observation arms, respectively. A minimization approach [35] will be applied to ensure balanced assignment to each treatment arm by: 1) Initial treatment with surgery vs. radiation therapy; 2) Prior hormonal therapy vs. no prior hormonal therapy; and 3) PSADT $<6$ mos vs. 6-14.9 mos. The on-study date for protocol entry will be the day that the study subject is randomized. Patients will be re-evaluated for radiographic response 6 months after randomization. Trial radiologists evaluating for treatment responses will be blinded to the treatment group and treatment specifics. The trial radiologists and the principal investigator will be unblinded only if a patient progresses at any time during the study.

\section{Interventions}

Eligibility work-up will include a complete blood count, serum chemistries, PSA, and radiographic studies (of involved sites) and bone scan. Subjects who meet eligibility criteria and qualify for enrollment will be stratified and randomized (Fig. 1).

The following is a detailed outline, after randomization, involving each study arm:

1. Observation Arm: Active Clinical Surveillance

a. Defined with 3 time points, involving the following:

Day 1

- Standard blood tests [complete blood count with differential ( $\mathrm{CBC}$ w/Diff), lactate dehydrogenase (LDH), serum chemistry, and PSA evaluation]

- Research laboratory tests: CAPP-Seq, rectal swab, and Color Test

Day 90

- Physician examination, medical history, medication review, performance status, quality of life (QoL) and adverse event (AE) evaluations

- Standard blood tests (CBC w/Diff, LDH, serum chemistry, testosterone, and PSA evaluation)

- Research blood tests: CAPP-Seq

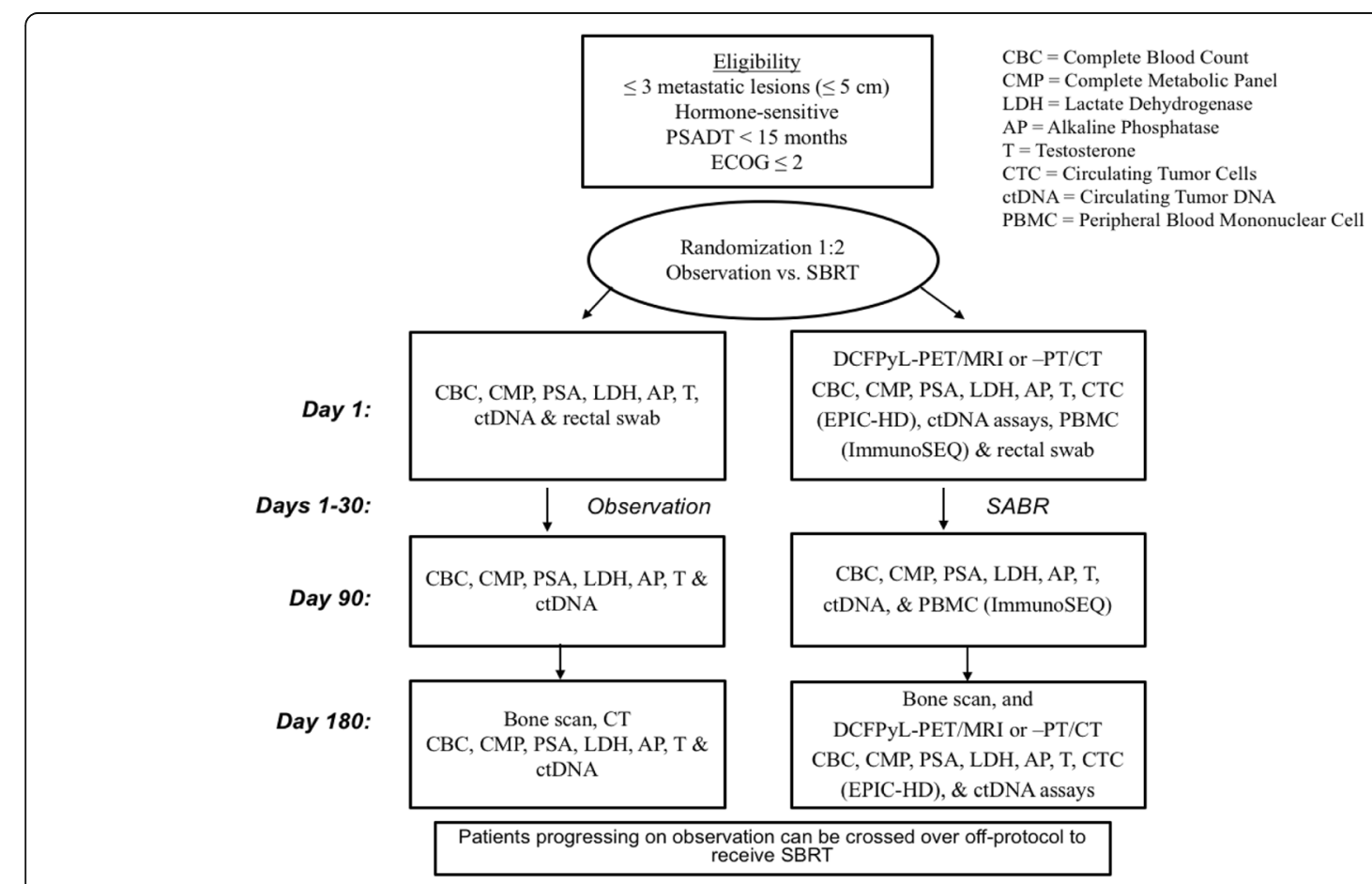

Fig. 1 ORIOLE Study Schema. Subjects who meet eligibility criteria and qualify for enrollment will be stratified and randomized as demonstrated 
Day 180

- Physician examination, medical history, medication review, performance status, QoL and AE evaluations

- Standard blood tests (CBC w/Diff, LDH, serum chemistry, testosterone and PSA evaluation),

- Research blood tests: CAPP-Seq

- Imaging: bone scan and CT/MRI

\section{SABR Arm:}

a. Defined with 3 time points, involving the following: Day 1

- Standard blood tests (CBC w/Diff, LDH, serum chemistry, and PSA evaluation) Research laboratory tests: EPIC HD-CTC, ImmunoSEQ, CAPP-Seq, rectal swab, and Color Test

- Imaging: ${ }^{18}$ F-DCFPyL PET/CT or PET/MRI

Day 90

- Physician examination, medical history, medication review, performance status, QoL and AE evaluations

- Standard blood tests (CBC w/Diff, LDH, serum chemistry, testosterone, and PSA evaluation)

- Research blood tests: ImmunoSEQ, CAPP-Seq Day 180

- Physician examination, medical history, medication review, performance status, QoL and AE evaluations

- Standard blood tests (CBC w/Diff, LDH, serum chemistry, testosterone, and PSA evaluation)

- Research blood tests: EPIC HD-CTC, CAPP-Seq

- Imaging: Bone scan and ${ }^{8}$ F-DCFPyL PET/CT or $\mathrm{PET} / \mathrm{MRI}$

b. Research Imaging

${ }^{18}$ F-DCFPyL PET/CT or PET/MRI images will be evaluated and compared to bone scan. However, additional sites(s) of suspected metastatic disease detected using ${ }^{18}$ F-DCFPyL will not be considered for treatment by SABR or undergo further evaluation. The results of PSMA-targeted PET imaging will not be made available to the treating physicians until after the trial is completed and these results will not be made available to the patient.

c. SBRT/SABR Planning and Dosage:

- CT- and/or MRI-simulation will be performed with fabrication of a radiation therapy immobilization device custom-made for each patient. The treating radiation oncologist will identify the location of the tumor. Gross tumor volume (GTV) delineation will be performed with a diagnostic radiologist on sequential axial computed tomography images. A radiosurgical treatment plan will then be developed based on tumor geometry and location. The clinical tumor volume (CTV) will equal the GTV. The dose will be prescribed to the minimal isodose line that completely covers the planning target volume (PTV), defined as CTV plus a variable (up to 5 $\mathrm{mm}$ ) margin. Adjacent normal structures, including but not limited to the heart, esophagus, aorta, spinal cord, kidneys, rectum, bowel, liver, and stomach, within $5 \mathrm{~cm}$ of the CTV will be identified for the purpose of limiting incidental radiation to these structures.

- In addition, prior to treatment delivery, a fourdimensional cone beam CT study will be performed on individual patients to assess respiration and determine targeting accuracy for tumors that may be subject to respiratory motion such as those in the bones of the thorax. If tumor motion is greater than $5 \mathrm{~mm}$, the PTV will be expanded accordingly.

- SABR will be delivered in 1 to 5 fractions, and the dose and fractionation schedule will depend on the size and location of the lesion and the surrounding normal tissue constraints in accordance with AAPM Task Group 101 recommendations [36].

- Within three weeks of the initial treatment planning imaging study, SABR will be administered using image-guidance. During treatment, real time cone beam CT images of the patient's body site of interest will be obtained. Cone beam CT will be obtained immediately prior to treatment and will be repeated until the treatment shift, required to align the planning CT and the cone beam CT performed on the day of treatment, is within tolerance for the body site.

\section{d. Early Stopping Guidelines}

- Site-specific grade 4/5 toxicity will be monitored in the SABR arm. If it becomes evident that the proportion of grade $4 / 5$ toxicity at specific sites convincingly exceeds $20 \%$, the study will be halted for a safety consultation. Specifically, we will apply a Bayesian toxicity monitoring rule that suspends the enrollment for a posterior probability $\geq 75 \%$ of toxicity being larger than $20 \%$. The monitoring rule uses Beta $(0.5,5.5)$ as prior distribution, meaning our prior guess of the proportion of toxicity is $8.3 \%$ with a $90 \%$ chance that this proportion is $0.04 \%-30.6 \%$.

Follow-Up:

- Patients will be followed from Day 1 to Day 180. 
- All AEs and serious adverse events (SAEs) are recorded on source documents. The investigator will follow up on all AEs and SAEs until the events have subsided, returned to baseline or, in case of permanent impairment, until the condition stabilizes.

\section{Stastical analysis \\ Sample size and accrual}

The primary endpoint will be progression of disease at 6 months. Historical data on this patient population indicate that $>80 \%$ would show progression, within a 6 -month period without treatment, and thus this is the progression rate we expect in subjects in the control/observation arm [37-39]. We hypothesize that SABR will reduce progression at 6 months by 50\% [40]. A sample size using a 2:1 randomization scheme of 36 in the SABR arm and 18 patients in the control group will provide $85 \%$ power to detect a decrease in relapse rate from $80 \%$ to $40 \%$ with a type I error $=0.05$ using one-sided Fisher's exact test. Thus, we will accrue a total of 54 patients. Patients withdrawing within one month of enrollment or prior to day 1 of SABR or observation will be replaced.

\section{Data analysis}

\subsubsection{Analysis of primary objective}

- A minimization approach [35] will be applied to ensure balanced assignment to each treatment arm by: 1 ) Initial treatment with surgery vs. radiation therapy; 2) Prior hormonal therapy vs. no prior hormonal therapy; and 3) PSADT <6 mos vs. 6-14.9 mos. Baseline PSA level is defined as that measured Day 1 following randomization.

- The primary clinical outcome will be the proportion of patients who have progressed after 6 months from randomization. For each arm, we will calculate the proportion of patients who have progressed and extract $95 \%$ confidence intervals. If a patient has withdrawn from the study before 6 months, they will be considered to have progressed.

- Progression will be a composite endpoint defined from the Prostate Cancer Working Group 2 (PCWG2) criteria for mCRPC [38] and our previous trials in a population of men with biochemical failure without metastases [37-39]. Progression will be defined as either: 1) a $\geq 25 \%$ increase in PSA from nadir (and by $\geq 2 \mathrm{ng} / \mathrm{mL}$ ), requiring confirmation $\geq 4$ weeks later (PCWG2 criteria); and/or, 2) clinical/ radiographic-progression defined as symptomatic progression (worsening disease-related symptoms or new cancer-related complications), or radiologic progression (on CT scan: $\geq 20 \%$ enlargement in sum diameter of soft-tissue target lesions [RECIST 1.1 criteria]; on bone scan: $\geq 1$ new bone lesions), initiation of ADT or death due to any cause, whichever occurs first. Death is considered a severe adverse event here.

- We will compare the proportion of patients who have progressed in the observation and SABR arms using Fisher's exact test. The analysis population includes all randomized subjects based on intentionto-treat.

\subsubsection{Analysis of secondary objectives}

- For safety analysis, adverse events will be summarized by type and grade.

- Hazard rate estimates and 95\% confidence intervals as well as Kaplan-Meier (KM) estimates will be calculated for PFS, ADT-FS, TTLP and TTDP. The median PFS, ADT-FS, TTLP and TTDP will be reported.

- Each metastatic lesion will be considered a target lesion and independently evaluated for response using RECIST 1.1 or bone scan evaluation criteria below. The lesion will be coded as locally controlled if it is considered stable radiographic disease or if there is evidence of a partial or complete response. Local control assessment will start at three months following randomization and continuous assessment will be pursued during the follow-up period. The proportion of locally controlled lesions will be estimated using generalized estimating equations.

- QoL will be assessed using the Brief Pain Inventory form. An overall score will be calculated pre-treatment and at the time of the $2^{\text {nd }}$ radiologic reassessment. The change in score will be evaluated by paired $t$-test.

\section{Response criteria}

a. Evaluation of Target Lesions and PSA Response

- Complete Response (CR): Disappearance of all target lesions and PSA below baseline

- Partial Response (PR): At least a 30\% decrease in the sum of the longest diameter (LD) of target lesions, taking as reference the baseline sum OR at least $1 / 3$ of lesions are stable or resolved by bone scan AND PSA below baseline

- Progressive Disease (PD): At least a 20\% increase in the sum of the LD of target lesions, taking as reference the smallest sum LD recorded since treatment initiation OR the appearance of $\geq 1$ new lesion(s) by bone scan OR PSA $\geq 25 \%$ above nadir or $>50 \mathrm{ng} / \mathrm{ml}$.

- Stable Disease (SD): Neither sufficient shrinkage to qualify for PR nor sufficient increase to qualify for $\mathrm{PD}$, taking as reference the smallest sum LD since 
treatment initiation OR PSA $\geq$ baseline but not $\geq 25 \%$ above nadir and $<50 \mathrm{ng} / \mathrm{ml}$.

- Complete Response (CR): Disappearance of all target lesions and PSA below baseline

- Partial Response (PR): At least a 30\% decrease in the sum of the longest diameter (LD) of target lesions, taking as reference the baseline sum OR at least $1 / 3$ of lesions are stable or resolved by bone scan AND PSA below baseline

- Progressive Disease (PD): At least a 20\% increase in the sum of the LD of target lesions, taking as reference the smallest sum LD recorded since treatment initiation OR the appearance of $\geq 1$ new lesion(s) by bone scan OR PSA $\geq 25 \%$ above nadir or $>50 \mathrm{ng} / \mathrm{ml}$.

- Stable Disease (SD): Neither sufficient shrinkage to qualify for PR nor sufficient increase to qualify for $\mathrm{PD}$, taking as reference the smallest sum LD since treatment initiation OR PSA $\geq$ baseline but not $\geq 25 \%$ above nadir and $<50 \mathrm{ng} / \mathrm{ml}$.

b. Evaluation of Best Overall Response: The best overall response is the best response recorded between treatment initiation and disease progression/recurrence

c. Duration of Response: Response will be defined as evidence of $\mathrm{CR}$, PR, or stable disease.

- Duration of CR or PR: The duration of CR or PR will be recorded from the date criteria for CR or PR are first met until the first date current or progressive disease is objectively documented (taking as reference for progressive disease the smallest measurements recorded since treatment initiation).

- Duration of Stable Disease: Stable disease will be recorded until the criteria for progression are met, taking as reference the smallest measurements recorded since the treatment started.

\section{Discussion}

The standard treatment options for metastatic hormone sensitive prostate cancer have remained unchanged for many years involving principally hormonal therapy. However, hormonal therapy can have troublesome side effects and any effort to delay the start of hormonal therapy would be an advantage to the patient. Radiation treatment was historically not given at high enough doses to metastases to provide durable local control. SABR is highly targeted radiation, delivered in a dose-intensive fashion in 1 to 5 fractions, which has been shown to be very effective on bone and soft tissue metastases. This phase II randomized study will compare SABR to observation with respect to progression of disease, freedom from hormonal therapy, and other relevant clinical endpoints. Simultaneously, a unique perspective on the impact of SABR on the biology of the oligometastatic state will be obtained through correlation of clinical response with measures of tumor burden, hematologic dynamics of metastasis, and immunologic response. Finally, the pursuit of patientcentered, personalized approaches to treatment will be furthered through investigation of targeted imaging and genomic susceptibility characterization.

\section{Abbreviations}

ADL: Activities of daily living; ADT: Androgen deprivation therapy; AE: Adverse event; BID: Twice daily; BSA: Body surface area; CBC: Complete blood count; Cl: Confidence interval; CMAX: Maximum concentration of drug; CNS: Central nervous system; CR: Complete response; CRF: Case report/Record form; CRPC: Castration-Resistant Prostate Cancer; CTC: Circulating Tumor Cell; CTCAE: Common Terminology Criteria for Adverse Events; DLT: Dose Limiting Toxicity; DSMB: Data Safety Monitoring Board; ECG: Electrocardiogram; Gl: Gastrointestinal; Hgb: Hemoglobin; HIV: Human Immunodeficiency Virus; HPF: High-power field; HTN: Hypertensions; IRB: Institutional Review Board; IV: Intravenous; LLN: Lower limit of normal; MTD: Maximum tolerated dose; OS: Overall survival; PD: Progressive diseased; PFS: Progression free survival; PLT: Platelet; PR: Partial response; PSADT: PSA Doubling Time; QD: Once daily; RECIST: Response evaluation criteria in solid tumors; RR: Response rate; SABR: Stereotactic ablative radiation therapy; SAE: Serious adverse event; SBRT: Stereotactic body radiation therapy; SD: Stable disease; TTP: Time to progression; ULN: Upper limit of normal; UNK: Unknown; WBC: White blood cell

\begin{abstract}
Acknowledgements
We would like to thank the following team members for their contribution to the success of this on-going trial: Helen Kim, Terry Caldwell, Christina Rodriguez, Shirl Dipasquale, Ashley Bruns, Jo Hurtt, Ruth Chamberlain, Barbara Squiller and Julie Ambrozak. The granting bodies had no role in the design or execution of the study; data collection, management, analysis, or interpretation of the data; preparation, review, or approval of the manuscript.
\end{abstract}

Funding

Grant Sponsors: NCl 1U01CA183031-01A1, PCF Young Investigator Award, and Movember-Prostate Cancer Foundation Challenge Award.

Availability of data and materials

The datasets used and/or analyzed during the current study are available from the corresponding author on reasonable request.

\section{Authors' contributions \\ Study conception: ME \& PTT. Study design: HW, ME \& PTT. Revision of the study: AR, SR, EA, CD, SG, SD, CP, KJP, ME \& PTT. Drafting manuscript: all authors. All authors have approved the manuscript.}

\section{Ethics approval and consent to participate}

This study will be carried out in compliance with the protocol and Good Clinical Practice, as described in: ICH Harmonized Tripartite Guidelines for Good Clinical Practice 1996; US 21 Code of Federal Regulations dealing with clinical studies (including parts 50 and 56 concerning informed consent and IRB regulations); and the Declaration of Helsinki, concerning medical research in humans (Recommendations Guiding Physicians in Biomedical Research Involving Human Subjects, Helsinki 1964, amended Tokyo 1975, Venice 1983, Hong Kong 1989, Somerset West 1996). The investigator agrees to adhere to the instructions and procedures described in it and thereby to adhere to the principles of Good Clinical Practice. Written informed consent are obtained from each patient before any study-specific procedure takes place. Participation in the study and date of informed consent patient are being documented appropriately in each patient's files. A Data Monitoring Committee is in place to monitor the trial. Data and safety monitoring oversight is conducted by the SKCCC at Johns Hopkins Safety Monitoring Committee. Per the SKCCC at Johns Hopkins Safety Monitoring plan, the CRO AQ will forward summaries of all monitoring reports to the Safety Monitoring Committee for review. 


\section{Competing interests}

MGP is a co-inventor on a US Patent covering ${ }^{18} \mathrm{~F}$-DCFPyL and as such is entitled to a portion of any licensing fees and royalties generated by this technology. This arrangement has been reviewed and approved by the Johns Hopkins University in accordance with its conflict of interest policies. MAG has served as a consultant to Progenics Pharmaceuticals, the licensee of ${ }^{18} \mathrm{~F}$-DCFPyL. The remaining authors declare no conflict of interest.

\section{Publisher's Note}

Springer Nature remains neutral with regard to jurisdictional claims in published maps and institutional affiliations.

\begin{abstract}
Author details
${ }^{1}$ Department of Radiation Oncology \& Molecular Radiation Sciences, The Sidney Kimmel Comprehensive Cancer Center, Johns Hopkins University School of Medicine, 1550 Orleans Street, CRB2 Rm 406, Baltimore, MD 21231, USA. ${ }^{2}$ Department of Medical Oncology, The Sidney Kimmel Comprehensive Cancer Center, Johns Hopkins University School of Medicine, Baltimore, MD, USA. ${ }^{3}$ The James Buchanan Brady Urological Institute and Department of Urology, Johns Hopkins University School of Medicine, Baltimore, MD, USA. ${ }^{4}$ The Russell H. Morgan Department of Radiology and Radiological Science, Johns Hopkins University School of Medicine, Baltimore, MD, USA ${ }^{5}$ Department of Radiation Oncology, Stanford University, Stanford, CA, USA. Department of Radiation Oncology, Thomas Jefferson University, Philadelphia, PA, USA.
\end{abstract}

Received: 8 February 2017 Accepted: 26 June 2017

Published online: 29 June 2017

\section{References}

1. Hellman S, Weichselbaum RR. Oligometastases. J Clin Oncol. 1995;13(1):8-10.

2. Schefter TE, Kavanagh BD, Timmerman RD, Cardenes HR, Baron A, Gaspar LE. A phase I trial of stereotactic body radiation therapy (SBRT) for liver metastases. Int J Radiat Oncol Biol Phys. 2005;62(5):1371-8.

3. Okunieff P, Petersen AL, Philip A, Milano MT, Katz AW, Boros L, Schell MC. Stereotactic body radiation therapy (SBRT) for lung metastases. Acta Oncol. 2006:45(7):808-17.

4. Lo SS, Fakiris AJ, Teh BS, Cardenes HR, Henderson MA, Forquer JA, Papiez L, McGarry RC, Wang JZ, Li K, Mayr NA, Timmerman RD. Stereotactic body radiation therapy for oligometastases. Expert Rev Anticancer Ther. 2009;9(5):621-35.

5. Milano MT, Katz AW, Muhs AG, Philip A, Buchholz DJ, Schell MC, Okunieff P. A prospective pilot study of curative-intent stereotactic body radiation therapy in patients with 5 or fewer oligometastatic lesions. Cancer. 2008;112(3):650-8

6. Milano MT, Katz AW, Schell MC, Philip A, Okunieff P. Descriptive analysis of oligometastatic lesions treated with curative-intent stereotactic body radiotherapy. Int J Radiat Oncol Biol Phys. 2008;72(5):1516-22.

7. Le QT, Loo BW, Ho A, Cotrutz C, Koong AC, Wakelee H, Kee ST, Constantinescu D, Whyte RI, Donington J. Results of a phase I dose-escalation study using single-fraction stereotactic radiotherapy for lung tumors. J Thorac Oncol. 2006;1(8):802-9.

8. Gibbs IC, Kamnerdsupai P, Ryu MR, Dodd R, Kiernan M, Chang SD, Adler JR. Image-guided robotic radiosurgery for spinal metastases. Radiother Oncol. 2007:82(2):185-90

9. Lo SS, Fakiris AJ, Abdulrahman R, Henderson MA, Chang EL, Suh JH, Timmerman RD. Role of stereotactic radiosurgery and fractionated stereotactic radiotherapy in pediatric brain tumors. Expert Rev Neurother. 2008;8(1):121-32.

10. Levine AM, Coleman C, Horasek S. Stereotactic radiosurgery for the treatment of primary sarcomas and sarcoma metastases of the spine. Neurosurgery. 2009;64(2 Suppl):A54-9.

11. Goodman KA, Wiegner EA, Maturen KE, Zhang Z, Mo Q, Yang G, Gibbs IC, Fisher GA, Koong AC. Dose-escalation study of single-fraction stereotactic body radiotherapy for liver malignancies. Int J Radiat Oncol Biol Phys. 2010;78(2):486-93.

12. Onishi $H$, Shirato $H$, Nagata $Y$, Hiraoka M, Fujino M, Gomi K, Niibe $Y$, Karasawa K, Hayakawa K, Takai Y, Kimura T, Takeda A, Ouchi A, Hareyama M, Kokubo M, Hara R, Itami J, Yamada K, Araki T. Hypofractionated stereotactic radiotherapy (HypoFXSRT) for stage I non-small cell lung cancer: updated results of 257 patients in a japanese multi-institutional study. J Thorac Oncol. 2007;2(7 Suppl 3):S94-100
13. Baumann P, Nyman J, Hoyer M, Wennberg B, Gagliardi G, Lax I, Drugge N, Ekberg L, Friesland S, Johansson KA, Lund JA, Morhed E, Nilsson K, Levin N, Paludan M, Sederholm C, Traberg A, Wittgren L, Lewensohn R. Outcome in a prospective phase II trial of medically inoperable stage I non-small-cell lung cancer patients treated with stereotactic body radiotherapy. J Clin Oncol. 2009;27(20):3290-6.

14. Timmerman R, Paulus R, Galvin J, Michalski J, Straube W, Bradley J, Fakiris A, Bezjak A, Videtic G, Johnstone D, Fowler J, Gore E, Choy H. Stereotactic body radiation therapy for inoperable early stage lung cancer. JAMA. 2010;303(11):1070-6. PMCID: PMC2907644

15. Videtic GM, Stephans K, Reddy C, Gajdos S, Kolar M, Clouser E, Djemil T. Intensity-modulated radiotherapy-based stereotactic body radiotherapy for medically inoperable early-stage lung cancer: excellent local control. Int J Radiat Oncol Biol Phys. 2010;77(2):344-9.

16. Fakiris AJ, McGarry RC, Yiannoutsos CT, Papiez L, Williams M, Henderson MA, Timmerman R. Stereotactic body radiation therapy for early-stage nonsmall-cell lung carcinoma: four-year results of a prospective phase II study. Int J Radiat Oncol Biol Phys. 2009;75(3):677-82.

17. Grills IS, Mangona VS, Welsh R, Chmielewski G, Mclnerney E, Martin S, Wloch J, Ye H, Kestin LL. Outcomes after stereotactic lung radiotherapy or wedge resection for stage I non-small-cell lung cancer. J Clin Oncol. 2010;28(6):928-35.

18. Wulf J, Haedinger U, Oppitz U, Thiele W, Mueller G, Flentje M. Stereotactic radiotherapy for primary lung cancer and pulmonary metastases: a noninvasive treatment approach in medically inoperable patients. Int J Radiat Oncol Biol Phys. 2004;60(1):186-96.

19. Hof H, Hoess A, Oetzel D, Debus J, Herfarth K. Stereotactic single-dose radiotherapy of lung metastases. Strahlenther Onkol. 2007;183(12):673-8.

20. Kim TH, Kim DY, Park JW, Kim YI, Kim SH, Park HS, Lee WJ, Park SJ, Hong EK, Kim CM. Three-dimensional conformal radiotherapy of unresectable hepatocellular carcinoma patients for whom transcatheter arterial chemoembolization was ineffective or unsuitable. Am J Clin Oncol. 2006;29(6):568-75.

21. Zhou ZH, Liu LM, Chen WW, Men ZQ, Lin JH, Chen Z, Zhang XJ, Jiang GL. Combined therapy of transcatheter arterial chemoembolisation and threedimensional conformal radiotherapy for hepatocellular carcinoma. Br J Radiol. 2007;80(951):194-201.

22. Seo DD, Lee HC, Jang MK, Min HJ, Kim KM, Lim YS, Chung YH, Lee YS, Suh DJ, Ko GY, Lee YJ, Lee SG. Preoperative portal vein embolization and surgical resection in patients with hepatocellular carcinoma and small future liver remnant volume: comparison with transarterial chemoembolization. Ann Surg Oncol. 2007;14(12):3501-9.

23. Kamel IR, Liapi E, Reyes DK, Zahurak M, Bluemke DA, Geschwind JF. Unresectable. hepatocellular carcinoma: Serial early vascular and cellular changes after transarterial chemoembolization as detected with MR imaging. Radiology. 2009;250(2):466-73

24. Cornford P, Bellmunt J, Bolla M, et al: EAU-ESTRO SIOG Guidelines on prostate cancer. Part II traetment of relapsing, metastatic, and castrationresistant prostate cancer. Eur Urol Adv DOI: 10.1016/j.eururo.2016.08.002.

25. Soloway MS, Hardeman SW, Hickey D, et al. Stratification of patients with metastatic prostate cancer based on extent of disease on initial bone scan. Cancer. 1988;61:195-202.

26. Singh D, Yi WS, Brasacchio RA, et al. Is there a favorable subset of patients with prostate cancer who develop oligometastases? Int J Radiat Oncol Biol Phys. 2004;58:3-10.

27. Schweizer MT, Zhou XC, Wang H, et al. Metastasis-free survival is associated with overall survival in men with PSA-recurrent prostate cancer treated with deferred androgen deprivation therapy. Ann Oncol. 2013;24:2881-6.

28. Sridharan S, Steigler A, Spry NA, et al. Oligometastatic bone disease in prostate cancer. patients treated on the TROG 03.04 RADAR trial. Radiother Oncol. 2016;121:98-102

29. Bostwick DG, Pacelli A, Blute M, Roche P, Murphy GP. Prostate specific membrane antigen expression in prostatic intraepithelial neoplasia and adenocarcinoma: a study of 184 cases. Cancer. 1998;82(11):2256-61.

30. Chang SS, Reuter VE, Heston WD, Gaudin PB. Comparison of anti-prostatespecific membrane antigen antibodies and other immunomarkers in metastatic prostate carcinoma. Urology. 2001;57(6):1179-83.

31. Sweat SD, Pacelli A, Murphy GP, Bostwick DG. Prostate-specific membrane antigen expression is greatest in prostate adenocarcinoma and lymph node metastases. Urology. 1998:52(4):637-40.

32. SEER Stat Fact Sheets: Colon and Rectum Cancer. Published 2015. Updated April 2016. Accessed 7 Aug 2016. http://seer.cancer.gov/statfacts/ html/colorect.html 
33. Color Genomics. (2016). Color genomics democratizes access to clinical-grade genetic testing worldwide. Published April 11 2016. Accessed January 312017. http://www.prnewswire.com/news-releases/color-genomics-democratizesaccess-to-clinical-grade-genetic-testing-worldwide-300248913.html.

34. Cleeland S. Pain Research Group. Copy right 1991. Accessed 17 Jan. 2017. http://prc.coh.org/pdf/BPI\%20Short\%20Version.pdf.

35. Vickers AJ. How to randomize. J Soc Integr Oncol. 2006:4:194-8.

36. Benedct SH, Yenice KM, Followill D, et al. Stereotactic body radiation therapy: the report of AAPM task group 101. Med Phys. 2010;37(8):5047-101.

37. Pound C, Partin A, Eisenberger M, Chan DW, Pearson JD, Walsh PC. Natural history of progression after PSA elevation following radical prostatectomy. JAMA. 1999;281:1591-7.

38. Antonarakis ES, Trock BJ, Feng Z, et al. The natural history of metastatic progression in men with PSA-recurrent prostate cancer after radical prostatectomy: 25-year follow-up. J Clin Oncol. 2009;27:15s. (suppl; abstr 5008

39. Decaestecker K, De Meerleer G, Lambert B, et al. Repeated stereotactic body radiotherapy for oligometastatic prostate cancer recurrence. Radiat Oncol. 2014;9:135.

40. Scher HI, Halabi S, Tannock I, et al. Design and end points of clinical trials for patients with progressive prostate cancer and castrate levels of testosterone: recommendations of the prostate cancer clinical trials working group. J Clin Oncol. 2008;26(7):1148-59.

\section{Submit your next manuscript to BioMed Central} and we will help you at every step:

- We accept pre-submission inquiries

- Our selector tool helps you to find the most relevant journal

- We provide round the clock customer support

- Convenient online submission

- Thorough peer review

- Inclusion in PubMed and all major indexing services

- Maximum visibility for your research

Submit your manuscript at www.biomedcentral.com/submit 